Artículo

\title{
Diseño y validación de instrumentos de medición
}

\author{
Design and validation of measurement instruments
}

\author{
Ana María Soriano Rodríguez* \\ asoriano@udb.edu.sv \\ ISSN 1996-1642, Editorial Universidad Don Bosco, año 8, No.13, Julio-Diciembre de 2014, pp. 19-40 \\ Recibido: 10 de julio de 2014. Aprobado: 8 de agosto de 2014
}

\section{Resumen}

El propósito de este artículo es explicar de forma sintética y lógica el diseño de un instrumento de medición. Se enfatiza principalmente la importancia de la validación como un proceso articulado que debe trascender de la confiabilidad a la validez, condiciones indispensables en todo proceso de medición en la investigación científica. Para facilitar la lectura, se incluyen referentes conceptuales con relación al diseño de los instrumentos y se ha estructurado el proceso del diseño y validación en cuatro fases. Cada una explica puntualmente los aspectos teóricos y los pasos operativos que deben ejecutarse en función de los jueces expertos y los procesos psicométricos, lo cuales permiten generar evidencias empíricas para la validación. Este artículo está dirigido a estudiantes de postgrados que realizan proyectos de investigación como requisitos de graduación y a profesionales de la academia que se inician en el campo investigativo de las áreas sociales y educativas.

Palabras clave: Instrumento, medición, validez, confiabilidad, consistencia interna y externa.

\begin{abstract}
In this article, the author approaches in a brief but logical way the design and validation of measurement instruments. She emphasizes the importance of validation as an articulating process that goes beyond validity and reliability as necessary conditions for rigorous scientific research. There is also a conceptual construct to support the design and validation. The approach includes four phases. Each one develops in detail the theoretical aspects, the operational steps that should be followed, the expert judgment and the psychometric processes which allow the generation of empirical evidences for validation. This article is addressed to those people at the university developing research as part of their postgraduate studies and those novice researchers from the academy.
\end{abstract}

Keywords: Instrument, measurement, validity, reliability, consistency

\footnotetext{
* Ana María Soriano Rodríguez es profesora investigadora del Instituto de Investigación y Formación de Pedagógica la Universidad Don Bosco y candidata a doctora en educación por la Universidad de Costa Rica.
}

Para citar este artículo: Soriano, A. M. (2014). Diseño y validación de instrumentos de medición. Diá-logos 14 , $19-40$. 


\section{Introducción}

Un instrumento de medida es una técnica o conjunto de técnicas que permitirán una asignación numérica que cuantifique las manifestaciones de un constructo que es medible solo de manera indirecta (Herrera, 1998). Los instrumentos de investigación son herramientas operativas que permiten la recolección de los datos; sin embargo, debe tenerse en cuenta que las prácticas de investigación sin una epistemología definida, se convierten en una instrumentalización de las técnicas (Sandín, 2003) por lo que todo instrumento deberá ser producto de una articulación entre paradigma, epistemología, perspectiva teórica, metodología y técnicas para la recolección y análisis de datos.

De acuerdo con Sandín (2003), un paradigma implica una forma de concebir e interpretar la realidad, involucra un modelo conceptual cuyo carácter normativo conducirá a los métodos y técnicas. Constituye una visión de mundo compartida por una comunidad y por ende posee un carácter socializador. Sautu (2003) define paradigma como la orientación general de una disciplina, el cual influye en la definición de los objetivos y la orientación metodológica en una investigación. "En términos de la próctica, el objetivo de la investigación dependerá del paradigma, la teoría general, los conceptos y proposiciones teóricas sustantivas y la metodología" (Sautu, 2003, p. 25).

En cuanto a los fundamentos epistemológicos en investigación, éstos se refieren a la concepción sobre el proceso de conocimiento que fundamenta la relación sujeto-objeto y el contexto en que está inmersa dicha relación. Es decir, el modelo que selecciona el investigador para relacionarse con lo investigado. GurdíanFernández (2007), Sautu (2003) y Sandín (2003) coinciden en la necesidad de una articulación entre paradigma, epistemología, teoría, metodología, técnicas e instrumentos en el diseño y ejecución de la investigación social.

Sautu (2003) define la teoría como el "conjunto de proposiciones lógicamente interrelacionadas del cual se derivan (siguen) implicaciones que se usan para explicar algunos fenómenos" (p. 27). Además, hace una clasificación entre una teoría general y teorías sustantivas, las cuales, impregnan la totalidad del diseño, la construcción del marco teórico que justifica la utilización de modelos estadísticos o estrategias cualitativas de análisis.

La teoría puede proveer al inicio de la investigación un enfoque o perspectiva que ubica el tipo de preguntas por hacer. La teoría también orienta sobre cómo los datos serán recolectados, organizados, analizados y presentados. Además, provee un llamado de atención para las acciones a tomar o cambios necesarios en el transcurso del estudio. Así, el investigador se guía sobre que hechos son importantes examinar y sobre los actores de la investigación (Creswell, 2009).

Diseño y validación de instrumentos 20. de medición
En cuanto a los instrumentos, estos se convierten en la herramienta concreta y operativa que facilitará al investigador la recolección de los datos, producto 
de una relación interdependiente entre paradigma, epistemología, teorías y metodologías; sin la definición, claridad, posicionamiento e interrelación de éstas no debería diseñarse un instrumento.

El siguiente gráfico ilustra como el paradigma seleccionado debe impregnar todo el proceso investigativo. Un error común al iniciarse en la investigación es fragmentar todos estos componentes y olvidar la función que la teoría tiene en la construcción del proceso metodológico y el diseño de los instrumentos. Una teoría bien definida y congruente con toda esta articulación orientará y facilitará la redacción de ítems que serán contrastados con la evidencia empírica.

llustración 1: Del paradigma a los instrumentos de investigación.

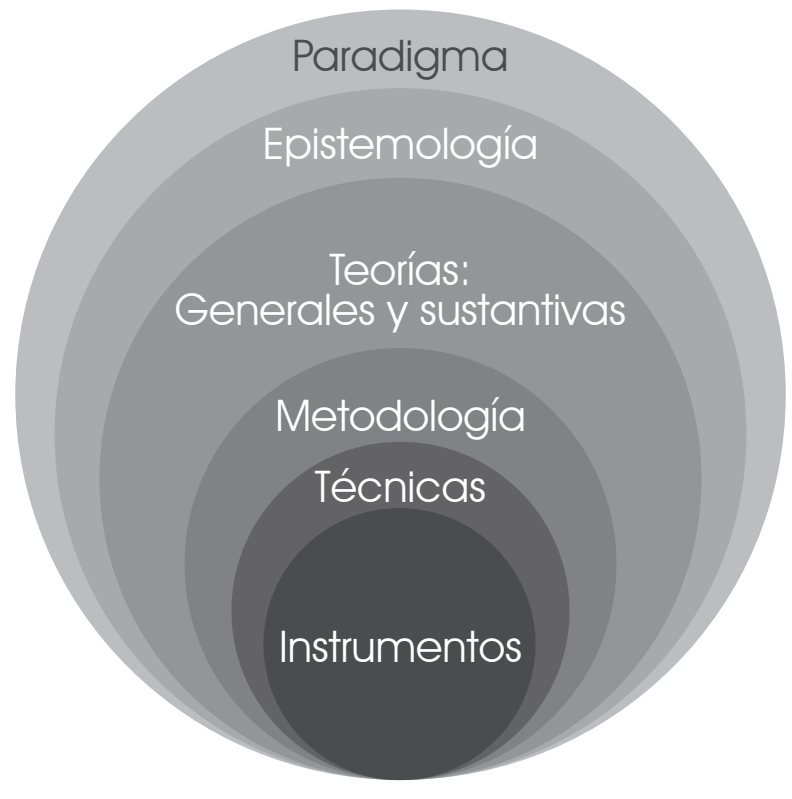

\section{Referentes conceptuales}

Para la elaboración de instrumentos debe tenerse claridad de los conceptos sobre constructo teórico, medición, confiabilidad y validez. Para Kerlinger (1988) un constructo es un concepto. Un concepto abstrae las generalizaciones de casos particulares; sin embargo, como constructo tiene el sentido adicional de haberse desarrollado o adoptado deliberadamente con un fin científico. El constructo es parte de los esquemas teóricos y está relacionado con otro.

Según Gras (1980) un constructo es la representación sobre algún aspecto sobre el objeto que será observado, medido y relacionado con otros constructos. Además, Briones (1998) establece que los constructos son medibles a través de sus manifestaciones externas, es decir, sus indicadores. Los constructos pueden ser definidos como propiedad subyacentes medidos solamente en forma indirecta, son definiciones mentales de los eventos de objetos los cuales pueden variar. 
De acuerdo con Carmines y Zeller (1987), la medición es un proceso que envuelve tanto consideraciones teóricas como empíricas. Desde el punto de vista empírico el enfoque está en las respuestas observables, ya sean a través de un cuestionario autoadministrado, observación directa o las respuestas obtenidas a través de una entrevista. En cuanto a lo teórico, el interés reside en los conceptos no observables (no medible directamente) representados en las respuestas dadas. La medición por tanto, se enfoca en esa relación entre los indicadores, que son las respuestas observadas y los conceptos no observables. Cuando la relación es significativamente fuerte, a través del análisis de los indicadores empíricos y los conceptos no observables, se establece inferencias entre éstos. Esto contribuye a evaluar la aplicabilidad empírica de las proposiciones teóricas, por el contrario, en el caso que la relación entre conceptos e indicadores son débiles, significa un modelo de medición inadecuado y cuyos resultado de una investigación realizada bajo ese modelo conlleva una falta de entendimiento del fenómeno social que se estudia.

Las principales propiedades de una medición son la confiabilidad y la validez (Carmines y Zeller, 1987). De acuerdo con Babbie (2000), la confiabilidad se refiere a que un objeto de estudio medido repetidamente con el mismo instrumento siempre dará los mismos resultados; sin embargo, la confiabilidad no garantiza, ni es sinónimo de exactitud. Un instrumento puede ser confiable, pero no necesariamente válido para una población en particular, o en el peor de los casos, que el instrumento haya sido manipulado para obtener ciertos resultados.

En cuanto a la validez de un instrumento, la definición tradicional se refería a la tautología es válido si mide lo que dice medir. Sin embargo, Messick $(1989,1996)$ argumenta que la definición tradicional es fragmentada e incompleta, por lo que, un instrumento será válido en cuanto que el grado de propiedad de las inferencias e interpretaciones producto de los resultados de un test incluya sus consecuencias sociales y éticas.

Es decir, Messick entiende la validez como un concepto unificado al cual le asigna un alto valor a cerca del cómo y para que los resultados del test son utilizados y sus consecuencias (por ejemplo como puede afectar un instrumento de medición en procesos de selección de personal, pruebas de admisión a centros educativos, test de conocimientos, etc.).

Alfaro y Montero (2013) establecen que la mayor contribución de Messick a la definición de validez es precisamente el concepto unitario que fuera adoptado formalmente por los Standards for Educational and Psychological Testing?', publicación conjunta de la AERA (American Educational Research Association)²,

Diseño y validación de instrumentos 22. de medición
1. Estándares para la medición educativa y psicológica

2. Asociación estadounidense de investigadores educativos 
APA (American Psychological Associaton) $)^{3}$ y NCME (National Council on Measurement in Education) 4 .

En lugar de clasificar los tipos de validez, Messick (1989) propone recolectar diferentes tipos de evidencias con base al uso y objetivos del instrumento, entre ellas la evidencia del contenido, del constructo y su valor predictivo. Además, debe tenerse en cuenta que la validez no es una propiedad intrínseca de los instrumentos, sino que dependerá del objetivo de la medición, la población y el contexto de aplicación, por lo que un instrumento puede ser válido para un grupo en particular pero no para otros. Debe considerarse que el proceso de validación es permanente y exige constantes comprobaciones empíricas, por lo que, no puede afirmarse contundentemente que una prueba es válida, sino que presenta un grado aceptable de validez para determinados objetivos y poblaciones.

De manera que, la confiabilidad es un hecho empírico, que se enfoca en probabilidad de obtener los mismos resultados al utilizar el mismo instrumento, mientras que la validez se cuestiona, que un instrumento sea válido para qué o en función de qué (Carmines y Zeller 1987). "Desde esta perspectiva, la validez psicométrica de un instrumento es solo una parte de la sistemática y rigurosa recolección de evidencia empírica, desde diferentes dimensiones, que debe emprenderse cuando se hace la pregunta: ¿Qué tan apropiadas son las inferencias generadas a partir de los puntajes de la prueba?" (Alfaro y Montero, 2013 p. 3), debe considerarse también que implicaciones éticas pueden llegar a tener los resultado de una prueba.

La validación de un instrumento no es un proceso acabado sino constante, al igual que todo proceso de la ciencia moderna, exige continuas comprobaciones empíricas. La validez no es un rasgo dicotómico, sino de grado, es decir que no se puede afirmar de manera concluyente que es una prueba es válida, sino que se puede afirmar de la prueba presenta ciertos grados de validez para ciertos usos concretos y determinadas poblaciones (Alfaro y Montero, 2013).

Teniendo en cuenta estos referentes conceptuales, la ilustración 2 esquematiza la secuencia lógica para diseñar un instrumento de investigación con fines de medición. Está dividido en cuatro fases, primero las consideraciones teóricas y objetivos de la investigación, segundo la validación de jueces expertos, tercero la selección de la muestra para la prueba piloto y la administración del instrumento y cuarto el proceso para la validación psicométrica.

llustración 2. Secuencia lógica para el proceso de diseño, redacción y validación de un instrumento. Fuente: Elaboración propia (2014).

3. Asociación Estadounidense de psicología

4. Concejo Nacional de Medición en Educación 

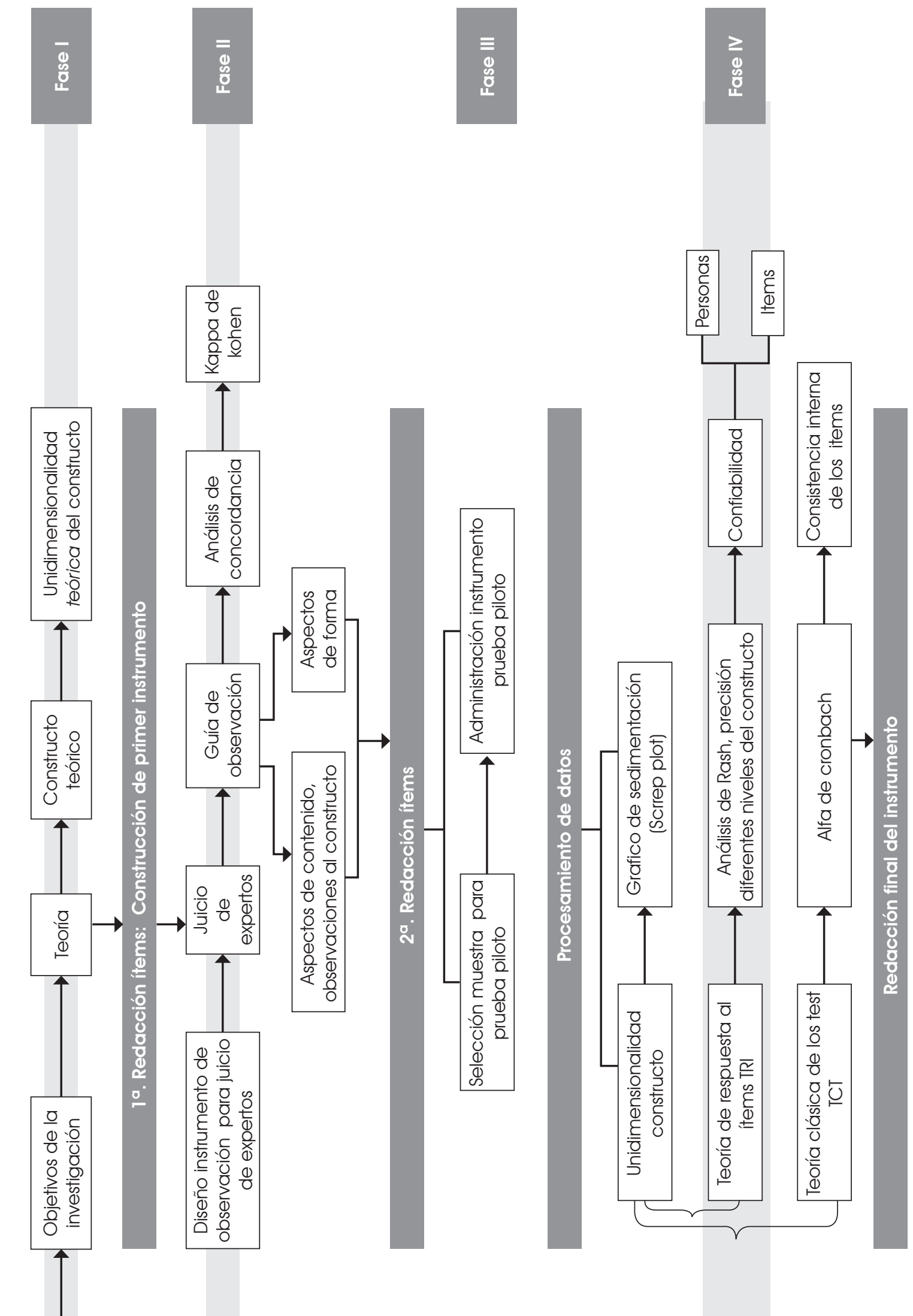


\section{Primera fase: Objetivos, teoría y constructo}

Al construir un instrumento debe tenerse claridad de los objetivos de la investigación y de las teorías generales y sustantivas que fundamentan y definen la opción teórica de la investigación. A partir de este posicionamiento, se definirá el constructo, el cual debe ser unidimensional.

"La dimensión es un aspecto o faceta especificable de un concepto" (Babbie 2000, p. 102). La formulación de la dimensión dependerá de cómo ha sido definido el constructo. Por ejemplo, si el posicionamiento teórico de una investigación sobre la inteligencia (dimensión) es la Teoría de Gardner sobre inteligencias múltiples deberá considerar como subdimensiones la inteligencia kinestésica, intrapersonal, musical, verbal, espacial, etc. (Abreu, 2012).

La unidimensionalidad se refiere a que la medición del instrumento (la escala o índice) se centrará en un atributo o característica. Un instrumento cuyo objetivo sea medir más de un atributo será considerado multidimensional. Constructos complejos como personalidad cuyas dimensiones pueden incluir inteligencia, autocontrol, etc., requieren de varias escalas unidimensionales. Se sugiere que los instrumentos de medición educativa sean unidimensionales, condición que deben cumplir para proceder a la validación psicométrica.

Toda erramienta deberá recolectar datos que están directamente relacionados con los fines de proyecto, obtener información que no conciernen a los objetivos de una investigación; además de incómodo para las personas que complementan los instrumentos, consumirá tiempo para su procesamiento y dificultarán el posterior análisis de datos.

Con base a los aspectos anteriores como punto de partida, es decir, objetivos, teorías, definición unidimensional del constructo, se procederá por parte del investigador a la redacción del primer set de ítems (llamados también reactivos).

\section{Segunda Fase: Validación juicio de expertos}

Al finalizar la primera redacción del instrumento se someterá a un juicio de expertos. Los expertos son personas cuya especialización, experiencia profesional, académica o investigativa relacionada al tema de investigación, les permite valorar, de contenido y de forma, cada uno de los ítems incluidos en la herramienta.

Los jueces deberán tener claridad de los objetivos y posicionamiento teórico de la investigación. Así, evaluarán, con base a los fines, constructo teórico y una guía de observación (ver ejemplo Cuadro No.1) la pertinencia de cada uno de los ítems o reactivos del instrumento.El cuadro 1 muestra un ejemplo de formato para una guía de observación para jueces expertos. Este puede ser adecuado de acuerdo con las exigencias del investigador, la investigación misma y los fines concretos del instrumento. Un formato definido garantiza que todos los 
jueces realizarán la misma observación bajo los mismos criterios a cada uno de los ítems. La carencia de un formato no permitiría realizar un posterior análisis de concordancia.

Cuadro 1. Ejemplo de formato para validación de instrumento por jueces expertos. Fuente: Elaboración propia (2014).

\begin{tabular}{|c|c|c|c|c|c|}
\hline \multicolumn{6}{|c|}{ Guía de observación para el instrumento de (...) } \\
\hline \multicolumn{6}{|l|}{ Objetivos (de esta guía) } \\
\hline \multicolumn{6}{|l|}{ Objetivos de la Investigación } \\
\hline \multirow{2}{*}{ Criterios a evaluar } & \multicolumn{2}{|c|}{ Ítem No. 1} & \multicolumn{2}{|c|}{ Ítem No.2 } & $(\ldots)$ \\
\hline & si & no & si & no & \\
\hline \multicolumn{6}{|l|}{ Claridad en la redacción } \\
\hline \multicolumn{6}{|l|}{ Coherencia interna } \\
\hline \multicolumn{6}{|l|}{ Sesgo (inducción a respuesta) } \\
\hline \multicolumn{6}{|l|}{ Redacción adecuada a la población en estudio } \\
\hline \multicolumn{6}{|l|}{ Respuesta puede estar orientada a la deseabilidad social } \\
\hline \multicolumn{6}{|l|}{ Contribuye a los objetivos de la investigación } \\
\hline \multicolumn{6}{|l|}{ Contribuye a medir el constructo en estudio } \\
\hline \multicolumn{6}{|l|}{ (..) } \\
\hline \multicolumn{6}{|l|}{$\begin{array}{l}\text { Observaciones a cada ítems, considerar si debe } \\
\text { eliminarse, modificarse, favor especificar) }\end{array}$} \\
\hline \multicolumn{6}{|l|}{ Consideraciones generales } \\
\hline \multirow{2}{*}{\multicolumn{4}{|c|}{ Las instrucciones orientan claramente para responder el cuestionario }} & si & no \\
\hline & & & & & \\
\hline \multicolumn{6}{|l|}{ La secuencia de los ítems es lógica } \\
\hline \multicolumn{6}{|l|}{ La cantidad de ítems es adecuada } \\
\hline \multicolumn{6}{|l|}{$(\ldots)$} \\
\hline \multicolumn{6}{|c|}{$\begin{array}{l}\text { Consideraciones finales (favor agregar observaciones que han sido consideradas } \\
\text { en este formato) }\end{array}$} \\
\hline \multicolumn{6}{|c|}{1.} \\
\hline \multicolumn{6}{|l|}{2.} \\
\hline \multicolumn{6}{|l|}{ Instrumento validad por: } \\
\hline Teléfono: & \multirow{2}{*}{\multicolumn{5}{|c|}{ Firma }} \\
\hline Correo electrónico: & & & & & \\
\hline \multicolumn{6}{|l|}{ Guía de observación para el instrumento de (...) } \\
\hline \multicolumn{6}{|l|}{ Objetivos (de esta guía) } \\
\hline \multicolumn{6}{|l|}{ Objetivos de la Investigación } \\
\hline Oitarion & Ítem & o. 1 & Íten & 0.2 & $(\ldots)$ \\
\hline & si & no & si & no & \\
\hline Claridad en la redacción & & & & & \\
\hline Coherencia interna & & & & & \\
\hline Sesgo (inducción a respuesta) & & & & & \\
\hline Redacción adecuada a la población en estudio & & & & & \\
\hline Respuesta puede estar orientada a la deseabilidad social & & & & & \\
\hline
\end{tabular}

Diseño y validación de instrumentos

26. de medición 


\begin{tabular}{|c|c|}
\hline Contribuye a los objetivos & \\
\hline Contribuye a medir el co & \\
\hline (..) & \\
\hline $\begin{array}{l}\text { Observaciones a ca } \\
\text { eliminarse, modif }\end{array}$ & \\
\hline Consideraciones genera & \\
\hline Las instrucciones orientan & no \\
\hline La secuencia de los ítems & \\
\hline La cantidad de ítems es C & \\
\hline$(\ldots)$ & \\
\hline $\begin{array}{l}\text { Consideraciones finales } \\
\text { en este formato) }\end{array}$ & \\
\hline 1. & \\
\hline 2. & \\
\hline Instrumento validad por: & \\
\hline Teléfono: & \\
\hline Correo electrónico: & \\
\hline
\end{tabular}

Las observaciones hechas por los jueces expertos deben ser sometidas a un análisis de concordancia, una opción es a través de la medida de Kappa de Cohen, la cual puede ejecutarse con el programa SPSS.

Precisa que, en cuanto al lenguaje y estilo de redacción del instrumento, se realice una validación exclusiva por parte de un grupo de jueces expertos, que procedan de una población similar a quien será administrado el instrumento. Por ejemplo, si una prueba será administrada a escolares de 5 años, lo ítems deben estar redactados de acuerdo con su edad, nivel educativo y condición socioeconómica, por lo que, además de un juez experto en pedagogía y un especialista en redacción que valide la prueba, deberá tenerse en cuenta a los escolares cuyas características correspondan a la muestra en estudio. La validación puede realizarse a través de grupos focales a los cuales se les preguntará sobre que interpretación dan a cada uno de los ítems.

Estos jueces darán certeza que el estilo de redacción de los ítems es comprendido por el grupo objetivo y por tanto asegura que las respuestas serán válidas. Las respuestas erróneas, en una prueba de conocimientos, puede ser el resultado de una errónea redacción de la pregunta y por tanto una equivocada interpretación por parte de quien es cuestionado. Debe tenerse claro que este proceso solamente asegura la lectura comprensiva de los ítems o reactivos por parte de la población objetiva, no es equivalente ni sustituye la prueba piloto cuya muestra debe ser seleccionada a través de una fórmula estadística.

El juicio de expertos permitirá al investigador mejorar los instrumentos en cuanto a los aspectos de contenido (dimensión teórica del constructo, selección de ítems, etc.) y los de forma y estilo (redacción de los ítems, comprensión, por parte 
de la población meta, etc.); sin embargo, este tipo de análisis es solamente una parte del proceso de validación de un instrumento y es preciso realizar las fases que posteriormente se detallan.

\title{
Tercera fase: Prueba piloto
}

De acuerdo con los resultados del análisis de concordancia entre los jueces expertos, se procede a una segunda redacción de ítems que conformarán el instrumento que será administrado para la prueba piloto, cuya muestra puede ser seleccionada a través de una muestra probabilística simple al azar.

Las características de la población para la prueba piloto deben ser similares a la muestra que se investigará. Se administra el instrumento bajo las mismas condiciones con las que se aplicará y posteriormente se procede al procesamiento de datos y análisis estadísticos descriptivos.

\section{Cuarta Fase: Validación Psicométrica}

Para explicar esta cuarta fase, se ha tomado como referencia un instrumento diseñado específicamente para estudiar el proceso de validación. Este se administró a 125 estudiantes del segundo semestre de una de las asignaturas del Curso de Formación Pedagógica de la Universidad Don Bosco, del total se omitieron las incompletas lo que hizo un total de 102 cuestionarios validos.

Cuadro 2. Ejemplo de validación psicométrica. Fuente Elaboración propia (2011)

\author{
Cuestionario para prueba piloto \\ Universidad Don Bosco \\ Vicerrectoría de Estudios de Posgrados
}

\section{Evaluación Docente}

El presente instrumento busca evaluar las competencias didácticas y desempeño docente dentro del aula como fuera de ella en el nivel de posgrado. El instrumento está divido en dos parte igualmente importante, la fase presencial y la fase a distancia. Por favor proporcione su opinión con respecto al último curso recibido. En el caso de la fase a distancia, esta no está adscrita únicamente a una plataforma sino a la forma en que el profesor tutor lo condujo durante la fase no presencial.

Parte 1:

Evaluación de las estrategias didácticas implementadas por el docente durante la clase presencial

Indicaciones: Marque la frecuencia con que se dan cada una de las siguientes afirmaciones.

Diseño y validación de instrumentos 28. de medición 


\begin{tabular}{|c|c|c|c|c|c|c|}
\hline $\mathrm{N}^{\circ}$ & El o la docente: & Nunca & A veces & $\begin{array}{c}\text { A } \\
\text { menudo }\end{array}$ & $\begin{array}{l}\text { Muy a } \\
\text { menudo }\end{array}$ & Siempre \\
\hline 1. & $\begin{array}{l}\text { Informó sobre la planificación de la } \\
\text { asignatura. }\end{array}$ & & & & & \\
\hline 2. & $\begin{array}{l}\text { Informó sobre los objetivos de la } \\
\text { asignatura. }\end{array}$ & & & & & \\
\hline 3. & $\begin{array}{l}\text { Informó sobre las actividades y } \\
\text { criterios de evaluación. }\end{array}$ & & & & & \\
\hline 4. & $\begin{array}{l}\text { Desarrolló a lo largo del curso clases } \\
\text { expositivas. }\end{array}$ & & & & & \\
\hline 5. & $\begin{array}{l}\text { Promovió la participación del alumno } \\
\text { en clase. }\end{array}$ & & & & & \\
\hline 6. & $\begin{array}{l}\text { Promovió el diálogo entre estudiantes } \\
\text { para abordar temas tratados en } \\
\text { clase. }\end{array}$ & & & & & \\
\hline 7. & $\begin{array}{l}\text { Ofreció retroalimentación a los } \\
\text { comentarios de los estudiantes. }\end{array}$ & & & & & \\
\hline 8. & $\begin{array}{l}\text { Preguntó sobre las conocimientos } \\
\text { previos de los estudiantes } \\
\text { relacionadas con las asignaturas. }\end{array}$ & & & & & \\
\hline 9. & Promovió el trabajo en equipo. & & & & & \\
\hline 10. & $\begin{array}{l}\text { Orientó el trabajo en equipo y } \\
\text { colaborativo. }\end{array}$ & & & & & \\
\hline 11. & $\begin{array}{l}\text { Promovió el debate, discusión o } \\
\text { interacción entre estudiantes. }\end{array}$ & & & & & \\
\hline 12. & $\begin{array}{l}\text { Orientó el debate y discusión entre } \\
\text { estudiantes. }\end{array}$ & & & & & \\
\hline 13. & Promovió el trabajo individual. & & & & & \\
\hline 14. & Orientó el trabajo individual. & & & & & \\
\hline 15. & $\begin{array}{l}\text { Asignó a grupo de estudiantes } \\
\text { exposiciones grupales para el } \\
\text { desarrollo de un tema. }\end{array}$ & & & & & \\
\hline 16. & $\begin{array}{l}\text { Retroalimentó las exposiciones } \\
\text { grupales por parte de los estudiantes. }\end{array}$ & & & & & \\
\hline 17. & $\begin{array}{l}\text { Fomentó el trabajo colaborativo entre } \\
\text { los estudiantes. }\end{array}$ & & & & & \\
\hline 18. & $\begin{array}{l}\text { Permitió un balance entre el trabajo } \\
\text { individual y grupal. }\end{array}$ & & & & & \\
\hline 19. & $\begin{array}{l}\text { Aplicó al menos una técnica } \\
\text { o dinámica para generar la } \\
\text { participación o debate entre } \\
\text { estudiantes. }\end{array}$ & & & & & \\
\hline 20. & Presentó estudios de casos. & & & & & \\
\hline 21. & $\begin{array}{l}\text { Propició el análisis y crítica de estudio } \\
\text { de casos. }\end{array}$ & & & & & \\
\hline 22. & $\begin{array}{l}\text { Brindó retroalimentación sobre } \\
\text { estudios de caso. }\end{array}$ & & & & & \\
\hline 23. & $\begin{array}{l}\text { Realizó demostraciones, ejemplos } \\
\text { orientadores para la realización de } \\
\text { tareas o actividades exaula. }\end{array}$ & & & & & \\
\hline 24. & Asignó lecturas complementarias & & & & & \\
\hline 25. & $\begin{array}{l}\text { Discutió, comentó en clase las } \\
\text { lecturas complementarias asignadas. }\end{array}$ & & & & & \\
\hline
\end{tabular}




\begin{tabular}{|c|l|l|l|l|l|l|}
\hline 26. & $\begin{array}{l}\text { Retroalimentó, al inicio de cada clase } \\
\text { los temas abordados el día anterior. }\end{array}$ & & & & & \\
\hline 27. & $\begin{array}{l}\text { Hizo uso de materiales audiovisuales } \\
\text { (videos, links en la web) que apoyaron } \\
\text { la explicación de temas. }\end{array}$ & & & & & \\
\hline 28. & $\begin{array}{l}\text { Hizo uso de diapositivas proyectadas } \\
\text { (power point u otros similares). }\end{array}$ & & & & & \\
\hline 29. & $\begin{array}{l}\text { Hizo uso efectivo de diapositivas } \\
\text { proyectas (legibles, uso de colores } \\
\text { adecuados). }\end{array}$ & & & & & \\
\hline 30. & $\begin{array}{l}\text { Hizo uso efectivo del pizarrón (legibles, } \\
\text { tamaño de letra adecuado). }\end{array}$ & & & & & \\
\hline 31. & $\begin{array}{l}\text { Ofreció los lineamientos claros para el } \\
\text { desarrollo de tareas exaulas. }\end{array}$ & & & & & \\
\hline
\end{tabular}

El primer análisis al cual debe someterse el instrumento es a la prueba de unidimensionalidad del constructo para ello se propone el análisis del grafico de sedimentación. Este puede ejecutarse a través del programa SPSS con un scree plot de Catell.

Gráfico 1: Gráfico de sedimentación de Cuestionario para Prueba Piloto.

\section{Scree Plot}

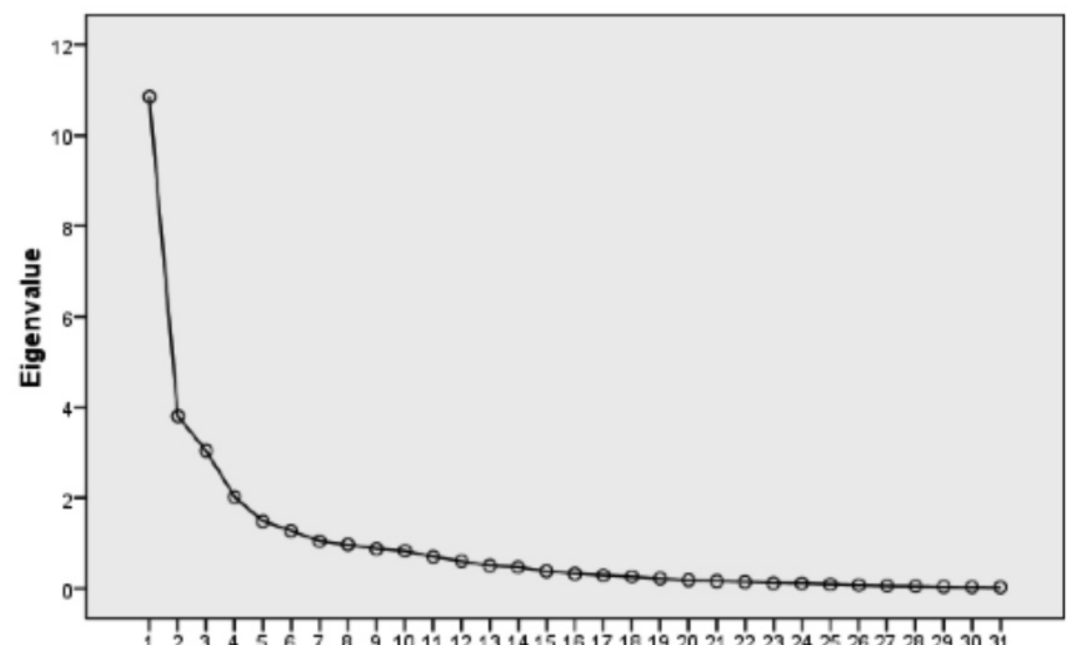

Component Number

De acuerdo con el Scree plot se podría evidenciar una unidimensionalidad en los instrumentos, dado que después del codo a nivel del punto 4 solamente le asciende un punto 11 aproximadamente. 
Tabla 1: Análisis de varranza de cuestionario prueba piloto.

Total Variance Explained

\begin{tabular}{|c|c|c|c|c|c|c|}
\hline \multirow{2}{*}{ Component } & \multicolumn{3}{|c|}{ Initial Eigenvalues } & \multicolumn{3}{|c|}{ Extraction Sums of Squared Loadings } \\
\hline & Total & $\%$ of Variance & Cumulative $\%$ & Total & $\%$ of Variance & Cumulative \% \\
\hline 1 & 10.857 & 35.022 & 35.022 & 10.857 & 35.022 & 35.022 \\
\hline 2 & 3.802 & 12.264 & 47.286 & 3.802 & 12.264 & 47.286 \\
\hline 3 & 3.039 & 9.802 & 57.088 & 3.039 & 9.802 & 57.088 \\
\hline 4 & 2.021 & 6.520 & 63.608 & 2.021 & 6.520 & 63.608 \\
\hline 5 & 1.484 & 4.786 & 68.394 & 1.484 & 4.786 & 68.394 \\
\hline 6 & 1.272 & 4.102 & 72.496 & 1.272 & 4.102 & 72.496 \\
\hline 7 & 1.045 & 3.372 & 75.868 & 1.045 & 3.372 & 75.868 \\
\hline 8 & .965 & 3.112 & 78.980 & & & \\
\hline 9 & .876 & 2.825 & 81.805 & & & \\
\hline 10 & .830 & 2.678 & 84.483 & & & \\
\hline 11 & .704 & 2.270 & 86.753 & & & \\
\hline 12 & .602 & 1.941 & 88.694 & & & \\
\hline 13 & .505 & 1.630 & 90.324 & & & \\
\hline 14 & .471 & 1.519 & 91.843 & & & \\
\hline 15 & .379 & 1.224 & 93.067 & & & \\
\hline 16 & .326 & 1.052 & 94.118 & & & \\
\hline 17 & .289 & .932 & 95.051 & & & \\
\hline 18 & .261 & .841 & 95.891 & & & \\
\hline 19 & .216 & .697 & 96.588 & & & \\
\hline 20 & .181 & .584 & 97.172 & & & \\
\hline 21 & .160 & .515 & 97.687 & & & \\
\hline 22 & .145 & .468 & 98.154 & & & \\
\hline 23 & .116 & .373 & 98.527 & & & \\
\hline 24 & .114 & .367 & 98.895 & & & \\
\hline 25 & .089 & .288 & 99.183 & & & \\
\hline 26 & .072 & .233 & 99.415 & & & \\
\hline 27 & .056 & .182 & 99.597 & & & \\
\hline 28 & .44 & .142 & 99.739 & & & \\
\hline 29 & .034 & .108 & 99.847 & & & \\
\hline 30 & .026 & .084 & 99.931 & & & \\
\hline 31 & .021 & .069 & 100.000 & & & \\
\hline
\end{tabular}

Extraction Method: Principal Component Analysis.

El porcentaje de varianza del primer componente es mayor a 20 y solamente el componente segundo es mayor a 10. Para que un instrumento sea considerado unidimensional el primer componente deberá ser mayor a 20 y el resto menor a 10, por lo tanto el instrumento analizado no puede considerarse multidimensional, dado que solamente el segundo componente es mayor a 10. A partir del tercer componente lo datos oscilan entre 9.28 hasta 0.069 .

Al contar con la evidencia de la unidimensionalidad del constructo se procede a la validación Análisis de confiabilidad y validez del instrumento. Sobre la confiablidad, se recuerda que esta se refiere a la consistencia interna del instrumento es decir la interrelación entre las preguntas que forman parte de la escala.

De acuerdo con la Teoría Clásica de los Test, la confiabilidad se define como el grado en que un instrumento construido por varios ítems presenta una alta correlación y miden consistentemente una muestra. Es decir, la dimensión 
considerada para el diseño del instrumento, sin embargo, debe recordarse que la confiabilidad no es criterio suficiente para determinar la validez de un instrumento.

Análisis Alpha de Cronbach

El Alpha de Cronbach permite realizar determinar la consistencia interna de los ítems y como esto se comportan entre ellos.

"El valor mínimo aceptable para el coeficiente alfa de Cronbach es 0.70; por debajo de ese valor la consistencia interna de la escala utilizada es baja. Por su parte, el valor máximo esperado es 0.90; por encima de este valor se considera que hay redundancia o duplicación. Varios ítems están midiendo exactamente el mismo elemento de un constructo; por lo tanto, los ítems redundantes deben eliminarse. Usualmente se prefieren valores de alfa entre 0.80 y 0.90. (Oviedo y Campos 2005, p. 577),

Además, de acuerdo con el análisis de Cronbach, la correlación de ítems (columna corrected Item -Total Correlation) con puntaje menor a 0.3 deberían ser eliminados.

\section{Primera prueba Alpha de Cronbach.}

Reliability Statistics

\begin{tabular}{|c|c|c|}
$\begin{array}{c}\text { Cronbach`s } \\
\text { Alpha }\end{array}$ & N of Items & $\begin{array}{l}\text { El alfa de Cronbach en } 0.93 \text { establece un alto } \\
\text { grado de confiabilidad interna del instrumento. }\end{array}$ \\
\cline { 1 - 2 } .923 & 31 &
\end{tabular}

Tabla.2. Primer Resultado Alpha de Cronbach

\begin{tabular}{|l|c|c|c|c|}
\hline & $\begin{array}{c}\text { Scale Mean } \\
\text { if Item } \\
\text { Deleted }\end{array}$ & $\begin{array}{c}\text { Scale } \\
\text { Variance } \\
\text { if Item } \\
\text { Deleted }\end{array}$ & $\begin{array}{c}\text { Corrected } \\
\text { Item-Total } \\
\text { Correlation }\end{array}$ & $\begin{array}{c}\text { Cronbach's } \\
\text { Alpha } \\
\text { if Item } \\
\text { Deleted }\end{array}$ \\
\hline $\begin{array}{l}\text { Informó sobre la planificación de la } \\
\text { asignatura. }\end{array}$ & 125.02 & 250.356 & .499 & .921 \\
\hline $\begin{array}{l}\text { Informó sobre los objetivos de la } \\
\text { asignatura. }\end{array}$ & 125.02 & 254.990 & .355 & .923 \\
\hline $\begin{array}{l}\text { Informó sobre las actividades y criterios } \\
\text { de evaluación. }\end{array}$ & 125.06 & 251.066 & .463 & .922 \\
\hline $\begin{array}{l}\text { Desarrolló a lo largo del curso clases } \\
\text { expositivas. }\end{array}$ & 125.31 & 254.990 & .217 & .924 \\
\hline $\begin{array}{l}\text { Promovió la participación del alumno en } \\
\text { clase. }\end{array}$ & 125.33 & 244.581 & .652 & .919 \\
\hline $\begin{array}{l}\text { Promovió el diálogo entre estudiantes } \\
\text { para abordar temas tratados en clase. }\end{array}$ & 125.45 & 241.438 & .656 & .919 \\
\hline
\end{tabular}




\begin{tabular}{|c|c|c|c|c|}
\hline $\begin{array}{l}\text { Ofreció retroalimentación a los } \\
\text { comentarios de los estudiantes. }\end{array}$ & 125.33 & 245.848 & .545 & .920 \\
\hline $\begin{array}{l}\text { Preguntó sobre las conocimientos previos } \\
\text { de los estudiantes relacionados con las } \\
\text { asignaturas. }\end{array}$ & 125.57 & 243.574 & .502 & .921 \\
\hline Promovió el trabajo en equipo. & 125.14 & 241.902 & .729 & .918 \\
\hline $\begin{array}{l}\text { Orientó el trabajo en equipo y } \\
\text { colaborativo. }\end{array}$ & 125.25 & 242.885 & .682 & .919 \\
\hline $\begin{array}{l}\text { Promovió el debate, discusión o } \\
\text { interacción entre estudiantes. }\end{array}$ & 125.53 & 236.687 & .736 & .918 \\
\hline $\begin{array}{l}\text { Orientó el debate y discusión entre } \\
\text { estudiantes. }\end{array}$ & 125.57 & 236.208 & .750 & .917 \\
\hline Promovió el trabajo individual. & 125.78 & 255.775 & .101 & .928 \\
\hline Orientó el trabajo individual. & 125.71 & 251.734 & .226 & .926 \\
\hline $\begin{array}{l}\text { Asignó a grupo de estudiantes } \\
\text { exposiciones grupales para el desarrollo } \\
\text { de un tema. }\end{array}$ & 125.41 & 239.849 & .564 & .920 \\
\hline $\begin{array}{l}\text { Retroalimentó las exposiciones grupales } \\
\text { por parte de los estudiantes. }\end{array}$ & 125.53 & 236.331 & 639 & .919 \\
\hline $\begin{array}{l}\text { Fomentó el trabajo colaborativo entre los } \\
\text { estudiantes. }\end{array}$ & 125.35 & 238.646 & .785 & .917 \\
\hline $\begin{array}{l}\text { permitio un balance entre el trabajo } \\
\text { grupal e individual }\end{array}$ & 125.69 & 247.465 & .376 & .923 \\
\hline $\begin{array}{l}\text { Aplicó al menos una técnica o dinámica } \\
\text { para generar la participación o debate } \\
\text { entre estudiantes. }\end{array}$ & 125.47 & 236.608 & .735 & .918 \\
\hline Presentó estudios de casos. & 125.63 & 242.771 & .485 & .921 \\
\hline $\begin{array}{l}\text { Propició el análisis y crítica de estudio de } \\
\text { casos. }\end{array}$ & 125.76 & 239.667 & .560 & .920 \\
\hline $\begin{array}{l}\text { Brindó retroalimentación sobre estudios } \\
\text { de caso. }\end{array}$ & 125.84 & 238.906 & .590 & .920 \\
\hline $\begin{array}{l}\text { Realizó demostraciones, ejemplos } \\
\text { orientadores para la realización de tareas } \\
\text { o actividades exaula. }\end{array}$ & 125.53 & 244.410 & .545 & .920 \\
\hline Asignó lecturas complementarias & 125.14 & 245.110 & .582 & .920 \\
\hline $\begin{array}{l}\text { Discutió, comentó en clase las lecturas } \\
\text { complementarias asignadas. }\end{array}$ & 125.16 & 243.341 & .694 & .919 \\
\hline $\begin{array}{l}\text { Retroalimentó, al inicio de cada clase los } \\
\text { temas abordados el día anterior. }\end{array}$ & 125.51 & 243.579 & .614 & .920 \\
\hline $\begin{array}{l}\text { Hizo uso de materiales audiovisuales } \\
\text { (videos, links en la web) que apoyaron la } \\
\text { explicación de temas. }\end{array}$ & 125.06 & 254.432 & .303 & .923 \\
\hline Hizo uso de diapositivas proyectadas & 125.06 & 253.402 & .321 & .923 \\
\hline $\begin{array}{l}\text { Hizo uso efectivo de diapositivas } \\
\text { proyectas (legibles, uso de colores } \\
\text { adecuados). }\end{array}$ & 125.22 & 250.468 & .457 & .922 \\
\hline $\begin{array}{l}\text { Hizo uso efectivo del pizarrón (legibles, } \\
\text { tamaño de letra adecuado). }\end{array}$ & 125.76 & 249.330 & .323 & .924 \\
\hline $\begin{array}{l}\text { Ofreció los lineamientos claros para el } \\
\text { desarrollo de tareas exaulas }\end{array}$ & 125.45 & 250.745 & .395 & .922 \\
\hline
\end{tabular}


Segunda prueba Alfa de Cronbach

Al eliminar el ítem Promovió el trabajo individual (ver

\begin{tabular}{|c|c|}
\hline $\begin{array}{c}\text { Cronbach`s } \\
\text { Alpha }\end{array}$ & $\begin{array}{c}\text { N of } \\
\text { Items }\end{array}$ \\
\hline .928 & 30 \\
\hline
\end{tabular}

Primera prueba), el alfa de Cronbach aumenta de 0.923 a 0928. Manteniéndose un total de 30 ítems. Obsérvese como los valores de las correlaciones de cada uno de los ítems cambia, lo cual afecta todo el instrumento.

Tabla No.3. Segundo Resultado Alpha de Cronbach.

Item - Total Statistics

\begin{tabular}{|c|c|c|c|c|}
\hline & $\begin{array}{l}\text { Scale Mean } \\
\text { if Item } \\
\text { Deleted }\end{array}$ & $\begin{array}{c}\text { Scale } \\
\text { Variance if } \\
\text { Item Deleted }\end{array}$ & $\begin{array}{l}\text { Corrected } \\
\text { Item-Total } \\
\text { Correlation }\end{array}$ & $\begin{array}{l}\text { Cronbach's } \\
\text { Alpha if Item } \\
\text { Deleted }\end{array}$ \\
\hline Informó sobre la planificación de la asignatura. & 121.22 & 244.369 & .525 & .926 \\
\hline Informó sobre los objetivos de la asignatura. & 121.22 & 249.359 & .362 & .928 \\
\hline Informó sobre las actividades y criterios de evaluación. & 121.25 & 245.222 & .481 & .927 \\
\hline Desarrolló a lo largo del curso clases expositivas. & 121.51 & 249.797 & .204 & .929 \\
\hline Promovió la participación del alumno en clase. & 121.53 & 238.588 & .677 & .924 \\
\hline $\begin{array}{l}\text { Promovió el diálogo entre estudiantes para abordar temas tratados } \\
\text { en clases }\end{array}$ & 121.65 & 235.597 & .673 & .924 \\
\hline Ofreció retroalimentación a los comentarios de los estudiantes & 121.53 & 240.172 & .555 & .926 \\
\hline $\begin{array}{l}\text { Preguntó sobre los conocimientos previos de los estudiantes } \\
\text { relacionados con las asignaturas. }\end{array}$ & 121.76 & 238.518 & .492 & .926 \\
\hline Promovió el trabajo en equipo. & 121.33 & 236.383 & .735 & .923 \\
\hline Orientó el trabajo en equipo colaborativo. & 121.45 & 237.161 & .695 & .924 \\
\hline Promovió el debate, discusión o interacción entre estudiantes. & 121.73 & 230.973 & .749 & .923 \\
\hline Orientó el debate y discusión entre estudiantes. & 121.76 & 230.875 & .751 & .923 \\
\hline Orientó el trabajo individual & 121.90 & 248.406 & .166 & .932 \\
\hline $\begin{array}{l}\text { Asignó a grupo de estudiantes exposiciones grupales para el desarrollo } \\
\text { de un tema }\end{array}$ & 121.61 & 234.102 & .575 & .925 \\
\hline Retroalimento las exposiciones grupales por parte de los estudiantes. & 121.73 & 230.498 & .654 & .924 \\
\hline Fomentó el trabajo colaborativo entre los estudiantes. & 121.55 & 233.280 & .786 & .923 \\
\hline Permitio un balance entre trabajo grupal e individual. & 121.88 & 243.709 & .325 & .929 \\
\hline $\begin{array}{l}\text { Aplicó al menos una técnica o dinámica para generar participación o } \\
\text { debate entre estudiantes. }\end{array}$ & 121.67 & 231.155 & .739 & .923 \\
\hline Presentó estudios de casos. & 121.82 & 237.771 & .474 & .927 \\
\hline Propició el análisis y critica de estudio de casos. & 121.96 & 234.414 & .557 & .926 \\
\hline Brindó retroalimentación sobre estudios de casos. & 122.04 & 233.464 & .593 & .925 \\
\hline $\begin{array}{l}\text { Realizó demostraciones, ejemplos orientadores para la realización de } \\
\text { tareas o actividades exaula. }\end{array}$ & 121.73 & 239.171 & .539 & .926 \\
\hline Asignó lecturas complementarias & 121.33 & 239.591 & .586 & .928 \\
\hline Discutió, comentó en clase las lecturas complementarias asignadas. & 121.35 & 237.577 & .710 & .924 \\
\hline $\begin{array}{l}\text { Retroalimento al inicio de cada clase los temas abordados el día } \\
\text { anterior. }\end{array}$ & 121.71 & 237.873 & .626 & .925 \\
\hline $\begin{array}{l}\text { Hizo uso de materiales audiovisuales (videos, links en la web) que } \\
\text { apoyaron la explicación de temas. }\end{array}$ & 121.25 & 248.627 & .317 & .928 \\
\hline Hizo uso de diapositivas proyectadas. & 121.25 & 247.479 & .339 & .928 \\
\hline $\begin{array}{l}\text { Hizo uso efectivo de diapositivas proyectadas (legibles, uso de colores } \\
\text { adecuados). }\end{array}$ & 121.41 & 244.561 & .478 & .927 \\
\hline Hizo uso efectivo del pizarrón (legibles, tamaño de letra adecuado) & 121.96 & 244.434 & .306 & .929 \\
\hline Ofreció los lineamientos claros para el desarrollo de tareas exaulas & 121.65 & 244.864 & .412 & .927 \\
\hline
\end{tabular}

\section{Tercera prueba}

Diseño y validación de instrumentos 34. de medición

\begin{tabular}{|c|c|}
\hline $\begin{array}{c}\text { Cronbach`s } \\
\text { Alpha }\end{array}$ & $\begin{array}{c}\text { N of } \\
\text { Items }\end{array}$ \\
\hline .932 & 29 \\
\hline
\end{tabular}

Al eliminar el ítem Orientó el trabajo individual (ver Primera prueba), el alfa de Crombach aumenta de 0.928 a 0932. Manteniéndose un total de 29 ítems. 
Tabla 4. Tercer resultado Alpha de Cronbach.

\begin{tabular}{|c|c|c|c|c|}
\hline & $\begin{array}{l}\text { Scale Mean } \\
\text { if Item } \\
\text { Deleted }\end{array}$ & $\begin{array}{c}\text { Scale } \\
\text { Variance if } \\
\text { Item Deleted }\end{array}$ & $\begin{array}{l}\text { Corrected } \\
\text { Item-Total } \\
\text { Correlation }\end{array}$ & $\begin{array}{l}\text { Cronbach's } \\
\text { Alpha if Item } \\
\text { Deleted }\end{array}$ \\
\hline $\begin{array}{l}\text { Informó sobre la planificación de la } \\
\text { asignatura. }\end{array}$ & 117.33 & 236.858 & .540 & .930 \\
\hline $\begin{array}{l}\text { Informó sobre los objetivos de la } \\
\text { asignatura. }\end{array}$ & 117.33 & 242.086 & .362 & .931 \\
\hline $\begin{array}{l}\text { Informó sobre las actividades y } \\
\text { criterios de evaluación. }\end{array}$ & 117.37 & 237.820 & .490 & .930 \\
\hline $\begin{array}{l}\text { Desarrolló a lo largo del curso clases } \\
\text { expositivas. }\end{array}$ & 117.63 & 242.929 & .187 & .933 \\
\hline $\begin{array}{l}\text { Promovió la participación del alumno } \\
\text { en clase. }\end{array}$ & 117.65 & 231.241 & .686 & .928 \\
\hline $\begin{array}{l}\text { Promovió el diálogo entre estudiantes } \\
\text { para abordar temas tratados en clase. }\end{array}$ & 117.76 & 228.459 & .676 & .928 \\
\hline $\begin{array}{l}\text { Ofreció retroalimentación a los } \\
\text { comentarios de los estudiantes. }\end{array}$ & 117.65 & 232.944 & .558 & .929 \\
\hline $\begin{array}{l}\text { Preguntó sobre las conocimientos } \\
\text { previos de los estudiantes relacionados } \\
\text { con las asignaturas. }\end{array}$ & 117.88 & 231.986 & .473 & .930 \\
\hline Promovió el trabajo en equipo. & 117.45 & 229.280 & .735 & .927 \\
\hline $\begin{array}{l}\text { Orientó el trabajo en equipo y } \\
\text { colaborativo. }\end{array}$ & 117.57 & 229.871 & .703 & .928 \\
\hline $\begin{array}{l}\text { Promovió el debate, discusión o } \\
\text { interacción entre estudiantes. }\end{array}$ & 117.84 & 223.936 & .750 & .926 \\
\hline $\begin{array}{l}\text { Orientó el debate y discusión entre } \\
\text { estudiantes. }\end{array}$ & 117.88 & 223.986 & .747 & .926 \\
\hline $\begin{array}{l}\text { Asignó a grupo de estudiantes } \\
\text { exposiciones grupales para el } \\
\text { desarrollo de un tema. }\end{array}$ & 117.73 & 226.815 & .582 & .929 \\
\hline $\begin{array}{l}\text { Retroalimentó las exposiciones } \\
\text { grupales por parte de los estudiantes. }\end{array}$ & 117.84 & 223.421 & .656 & .928 \\
\hline $\begin{array}{l}\text { Fomentó el trabajo colaborativo entre } \\
\text { los estudiantes. }\end{array}$ & 117.67 & 226.442 & .778 & .926 \\
\hline $\begin{array}{l}\text { permitio un balance entre el trabajo } \\
\text { grupal e individual }\end{array}$ & 118.00 & 237.941 & .280 & .933 \\
\hline $\begin{array}{l}\text { Aplicó al menos una técnica } \\
\text { o dinámica para generar la } \\
\text { participación o debate entre } \\
\text { estudiantes. }\end{array}$ & 117.78 & 223.933 & .746 & .926 \\
\hline Presentó estudios de casos. & 117.94 & 230.630 & .475 & .931 \\
\hline $\begin{array}{l}\text { Propició el análisis y crítica de estudio } \\
\text { de casos. }\end{array}$ & 118.08 & 227.083 & .565 & .929 \\
\hline $\begin{array}{l}\text { Brindó retroalimentación sobre } \\
\text { estudios de caso. }\end{array}$ & 118.16 & 225.995 & .605 & .929 \\
\hline $\begin{array}{l}\text { Realizó demostraciones, ejemplos } \\
\text { orientadores para la realización de } \\
\text { tareas o actividades exaula. }\end{array}$ & 117.84 & 231.975 & .542 & .929 \\
\hline Asignó lecturas complementarias & 117.45 & 232.250 & .594 & .929 \\
\hline $\begin{array}{l}\text { Discutió, comentó en clase las } \\
\text { lecturas complementarias asignadas. }\end{array}$ & 117.47 & 230.390 & .713 & .928 \\
\hline
\end{tabular}




\begin{tabular}{|l|c|c|c|c|}
\hline $\begin{array}{l}\text { Retroalimentó, al inicio de cada clase } \\
\text { los temas abordados el día anterior. }\end{array}$ & 117.82 & 230.761 & .626 & .928 \\
\hline $\begin{array}{l}\text { Hizo uso de materiales audiovisuales } \\
\text { (videos, links en la web) que apoyaron } \\
\text { la explicación de temas. }\end{array}$ & 117.37 & 241.068 & .331 & .932 \\
\hline Hizo uso de diapositivas proyectadas & 117.37 & 239.800 & .359 & .931 \\
\hline $\begin{array}{l}\text { Hizo uso efectivo de diapositivas } \\
\text { proyectas (legibles, uso de colores } \\
\text { adecuados). }\end{array}$ & 117.53 & 236.885 & .499 & .930 \\
\hline $\begin{array}{l}\text { Hizo uso efectivo del pizarrón (legibles, } \\
\text { tamaño de letra adecuado). }\end{array}$ & 118.08 & 237.697 & .291 & .933 \\
\hline $\begin{array}{l}\text { Ofreció los lineamientos claros para el } \\
\text { desarrollo de tareas exaulas }\end{array}$ & 117.76 & 237.449 & .421 & .931 \\
\hline
\end{tabular}

\section{Análisis de Rasch}

La segunda prueba de confiabilidad aplicada fue el análisis de Rasch (realizado con el software Winstep). Esta prueba presenta la ventaja que pueden analizarse simultáneamente la dificultad de los ítems en función de las personas.

Al realizar el análisis de Rasch la confiabilidad del instrumento marca 0.98 para los ítems y 0.87 para personas. El INFIT para cada uno de los ítems oscila entre 0.80 y 1.45 , con un promedio de 1.04. De acuerdo con el criterio de Prieto y Delgado (2003) los ítems que marquen un INFIT menor de 0.3 o mayor de 1.7, para muestras menores de 500 deben ser eliminados. (En este caso, la muestra fue de 125 alumnos).

Tabla 5. Modelo de Rasch.

INPUT: 102 PERSON 17 ITEM MEASURED: 102 PERSON 17 ITEM 76 CATS WINSTEPS 3.70.0.2 PERSON: REAL SEP:: 2.37 REL.: .85 . . ITEM: REAL SEP.: 2.57 REL.: .87 ITEM STATISTICS: MEASURE ORDER

Diseño y validación de instrumentos 36. de medición
Las principales interpretaciones de estos resultados es que el instrumento fue completado por 102 personas y que después de la depuración de ítems de 29 través se observa el número de ítems resultantes son 17. En la columna ítem $G$ (primera de derecha a izquierda) define los ítems validos para la prueba. 
Ítems finales de para rediseño de instrumento (1 7 en total de 29, columna Ítem G)

A1: Informó sobre la planificación de la asignatura.

A2: Informó sobre los objetivos de la asignatura

A3: Informó sobre las actividades y criterios de evaluación

A5: Promovió la participación del alumno en clase.

A6: Promovió el diálogo entre estudiantes para abordar temas tratados en clase.

A7: Ofreció retroalimentación a los comentarios de los estudiantes.

A10: Orientó el trabajo en equipo y colaborativo.

A12: Orientó el debate y discusión entre estudiantes.

A15: Asignó a grupo de estudiantes exposiciones grupales para el desarrollo de un tema.

A16: Retroalimentó las exposiciones grupales por parte de los estudiantes.

A19: Aplicó al menos una técnica o dinámica para generar la participación o debate entre estudiantes.

A22: Brindó retroalimentación sobre estudios de caso.

A23: Realizó demostraciones, ejemplos orientadores para la realización de tareas o actividades exaula

A24: Asignó lecturas complementarias

A25: Discutió, comentó en clase las lecturas complementarias asignadas.

A26: Retroalimentó, al inicio de cada clase los temas abordados el día anterior.

A29: Hizo uso efectivo de diapositivas proyectas (legibles, uso de colores adecuados).

Adviértase que el número de ítems se reduce considerablemente, por lo que al iniciar la redacción de ítems

Mapa territorial

Muestra que el instrumento resulta fácilmente comprensible por las personas que lo complementaron. La mayoría de estudiantes están arriba de los ítems. La desviación típica podría considerarse casi perfecta ya que hay personas tanto arriba como debajo de la media.

Las personas están arriba de la media, lo cual indica que los ítems son entendibles y que las personas lograron fácilmente interpretarlo. El análisis da como resultado 51 cuestionarios validos (complementados por 51 personas de 102) y 17 ítems resultantes de 29 previamente seleccionado con base al análisis del Alpha de Cronbach. 
Gráfica 2: Mapa territorial.

TABLE 1.0 BASE DE DATOS DEPURADO PERSONS 0.5 sav ZOU785WS.TXT Dec 19 17:56 2010 INPUT: 51 PERSON 17 ITEM MEASURED: 51 PERSON 17 ITEM 75 CATS WINSTEPS 3.70.0.2

- - - - - - - - - - - - - - - - - - - - - - - - - - - - - - - -

4

PERSON - MAP - ITEM $<$ more $>$ i $<$ rare $>$

$1203+$

$758 \mathrm{~T}$

36

43

8794

8

$9 \quad 15$

3436

$60 \quad 66 \quad 85 s^{\prime}$

2

1869

$\begin{array}{llllcc}26 & 32 & 49 & 77 & 83 & 100 \\ & & & & & 55\end{array}$

1

$\begin{array}{rllllllr}16 & 42 & 46 & 51 & 67 & 93 & 94 & 71 \\ & & & & & & & \end{array}$

0

$\begin{array}{llllll}2 & 22 & 53 & 73 & \text { A22 }\end{array}$

$2139 \quad 72 \quad 90$ IIS A12

A16 A6

A19
$+\mathrm{MA} 23$

$+\mathrm{MA} 23 \quad \mathrm{~A} 26$

I A10

A24 A29

IS A1 A25 A3

(2)

IT

$-1$

T I

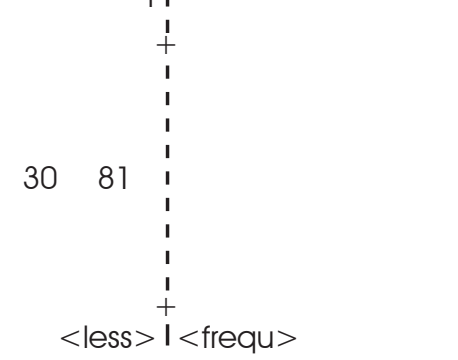

TABLE 1.1 BASE DE DATOS DEPURADO PERSONS 0.5 sav ZOU785WS.TXT DeC 19 17:56 2010 INPUT: 51 PERSON 17 ITEM MEASURED: 51 PERSON 17 ITEM 75 CATS WINSTEPS 3.70.0.2

\section{Consideraciones finales}

El diseño de instrumentos y sus correspondientes ítems, ya sea para evaluación o pruebas académicas deben pasar por todo el proceso anterior para asegurar

Diseño y validación de instrumentos 38. de medición que la información que se obtenga sea válida y permita una efectiva toma de decisiones. 
Los ítems deben tener como punto de partida un constructo teórico, respaldado por jueces expertos y análisis estadísticos que validen los instrumentos, de lo contrario, se estaría induciendo a ofrecer soluciones incongruentes o que puedan afectar a una población estudiantil o a docentes en el caso de evaluaciones.

Como educadores, al impartir clases de metodologías de la investigación tenemos la obligación de hacer suficiente énfasis a los estudiantes que el diseño de instrumentos no es un set de preguntas que seleccionan como producto de una lluvia de ideas, sino que requiere de un proceso riguroso que demuestren su validez empírica.

De la misma manera, al hacer referencia a otras investigaciones educativas deberíamos estar en la capacidad de cuestionar sus resultados en base al diseño de un instrumento. Estas preguntas servirán para orientarnos: ¿Qué constructo teórico se ha tomado como base para la construcción de los ítems? ¿Cuáles pruebas psicométricas aseguran la confiabilidad y validez de instrumento? ¿El constructo teórico es unidimensional? ¿Cuál fue el resultado del juicio de expertos? ¿Quiénes fueron los expertos? ¿Prueba piloto en contraste del juicio de experto? ¿A cuáles objetivos de la investigación contribuye este instrumento?.

Como investigadores educativos tenemos la obligación de diseñar los instrumentos con rigurosidad científica, caso contrario, no podrá plantearse los resultados de una investigación como válidos, así se haya administrado a una muestra representativa y el margen de error sea muy bajo. Especialmente investigaciones cuyos resultados impactarán en la toma de decisiones e implicaran selección o promoción de estudiantes o docentes.

\section{Referencias}

Abreu, J. (2012). Constructos, Variables, Dimensiones, Indicadores \& Congruencia. Daena: International Journal of Good Conscience, 7(3), 123-130. http:// www.spentamexico.org/v7-n3/7(3)123-130.pdf

Alfaro, K. y Montero, E. (2013). Aplicación del modelo de Rasch, en el análisis psicométrico de una pruebade diagnóstico en matemática. Revista digital Matemática, Educación e Internet 13(1). Oobtenido de http:// www.tec-digital.itcr.ac.cr/revistamatematica/ ARTICULOS_V13_N1_2012/ RevistaDigital_Montero_V13_n1_2012/index.html

Babbie, E. (2010). The Practice of Social Research. California: Wadsworth.

Briones, G. (1998). Métodos y técnicas de investigación para las ciencias sociales. México: Trillas.

Carmines, E. y Zeller, R. (1987). Reliability and Validity Assessment. USA: Sage

Creswell, J. (2009). Research Design: Qualitative, Quantitative, and Mixed Mothods Approaches. Estados Unidos: Sage.

Gras, A. (1980). Psicología experimental. Un enfoque metodológico. México: Trillas. 
Gurdián-Fernández, A. (2007). El Paradigma Cualitativo en la investigación Socio-educativa. San José: Print Center.

Herrera,A. (1998). Notas sobre Psicometría. Bogotá: Universidad Nacional de Colombia.

Kerlinger, F. (1988). Investigación del Comportamiento. Segunda Edición. México: McGraw-Hill.

Messick, S. (1989). Validity. en R. L. Linn (Ed.), Educational measurement (3rd ed., pp. 13-103). New York: Macmillan.

Messick, S. (1996). Standards-based score interpretation: Establishing valid grounds for valid inferences. Washington DC: Government Printing Office.

Oviedo, H. .y Campos, Aproximación al uso del coeficiente de Alfa de Cronbach. Revista Colombiana de Psiquiatría, 34(4), 572-580. http://www.redalyc. org/articulo.oa?id $=80634409$

Prieto, G. y Delgado A. (2003). Análisis de un test mediante el modelo de Rasch. Psicothema, 15(1), 94-100.

Sandín, M. (2003). Investigación Cualitativa en Educación: Fundamentos y tradiciones. España: McGrw-Hill

Sautu, R. (2003). Todo es teoría. Objetivos y métodos de investigación. Buenos Aires: Editorial Lumiere. 